\title{
The massive Wolf-Rayet binary WR 98 (WN7/WC+O8-9)
}

\author{
Roberto C. Gamen ${ }^{1}$ and Virpi S. Niemela ${ }^{2}$ \\ Facultad de Ciencias Astronómicas y Geofísicas, Universidad Nacional \\ de La Plata, Paseo del Bosque s/n, B1900FWA, La Plata, Argentina
}

\begin{abstract}
We present the discovery of $O B$ type absorption lines superimposed to the emission line spectrum, and the first double-lined orbital elements, for the massive Wolf-Rayet binary WR 98 (HDE 318016, WN7/C+O8-9), a spectroscopic binary in a circular orbit with a period of $47.825 \mathrm{~d}$. The semi-amplitudes of the orbital motion of the emission lines differ from line to line, indicating a mass ratio in the range $q \equiv M_{\mathrm{WR}} / M_{\mathrm{OB}}=1-1.8$.
\end{abstract}

\section{Introduction and observations}

WR 98 (HDE 318016, Tr27-105) is one of the few Wolf-Rayet stars showing both $\mathrm{N}$ and $\mathrm{C}$ emission lines (van der Hucht 2001), causing its classification WC7WN6 (Smith (1968), WN7+WC7 (van der Hucht et al. 1981), and WN8/WC7 (Smith et al. 1996). WR 98 was found to be a single-lined spectroscopic binary, with $P=47.8 \mathrm{~d}$ and with $\mathrm{N}$ and $\mathrm{C}$ emission lines moving in phase by Niemela (1991). We present here the results of a detailed radial-velocity (RV) analysis of optical spectral lines of WR 98 (Gamen \& Niemela, 2002), showing it to be a double-lined spectroscopic binary (SB2).

We have obtained a total of 69 blue optical spectrograms of WR 98 at CTIO in Chile (1980 - 84) and CASLEO ${ }^{3}$ in Argentina (1997-2001). The observations at CTIO were performed with the Cassegrain IT spectrograph on the $1 \mathrm{~m}$ Yale telescope, using photographic plates with fine grain emulsion as detector. The observations at CASLEO where obtained with the CCD Cassegrain spectrographs attached to the $2.15 \mathrm{~m}$ telescope. The photographic observations were digitized with a Grant machine at La Plata. The digital data were processed and measured with IRAF routines.

\section{Results}

Our RV analysis of the optical spectral lines of WR 98, using a long term database, confirms that this star is a binary system with $e=0$ and $P=47.825 \mathrm{~d}$ (see Figure 1). $\mathrm{N}$ and $\mathrm{C}$ emission lines in the spectrum move in phase, indicating

\footnotetext{
${ }^{1}$ Fellow of CONICET, Argentina

${ }^{2}$ Visiting Astronomer, CTIO, NOAO, operated by AURA, Inc. for NSF, USA. Member of Carrera del Investigador, CIC-BA, Argentina

${ }^{3}$ CASLEO is operated under agreement between CONICET, SeCyT, and the universities of La Plata, Córdoba and San Juan, Argentina
} 

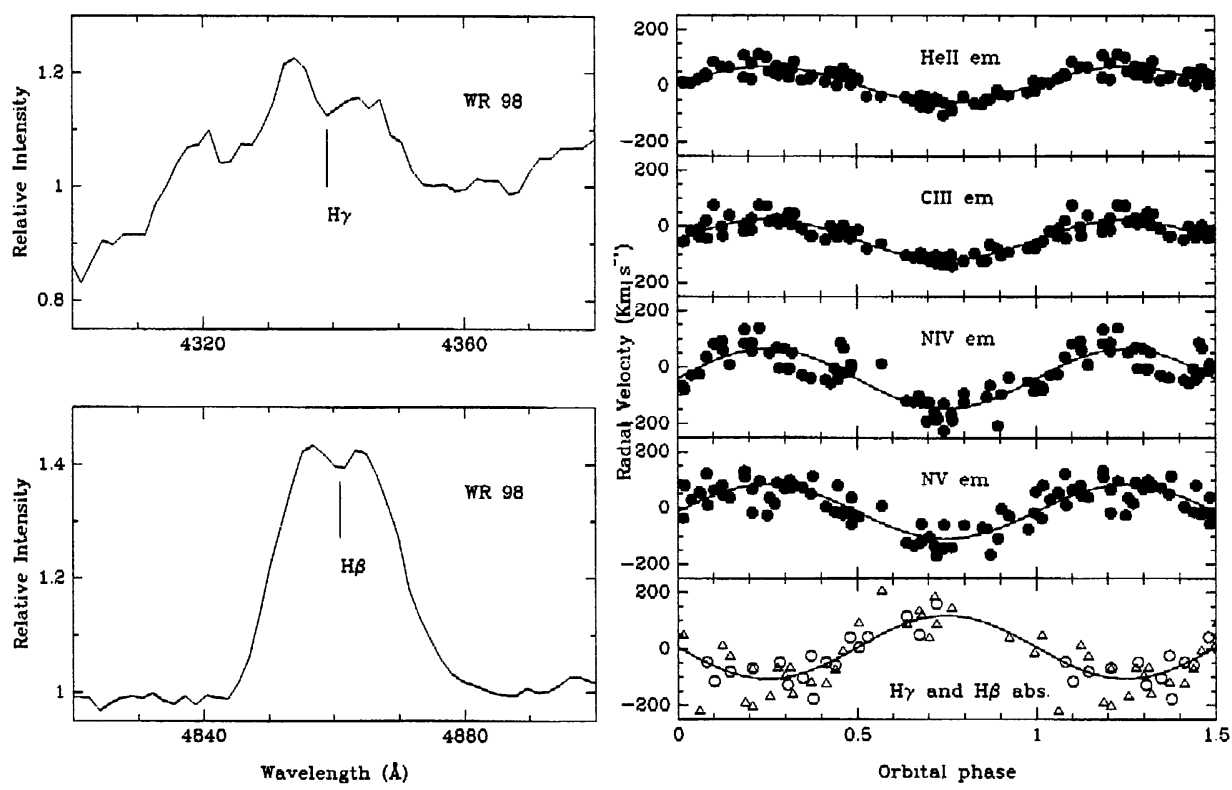

fral phase

Figure 1. Left: $\mathrm{H} \gamma$ and $\mathrm{H} \beta$ absorption in He II emission of WR 98. Right: RV variations of emission lines and absorption of $\mathrm{H} \gamma$ (circles) and $\mathrm{H} \beta$ (triangles) phased with $P=47.825 \mathrm{~d}$. Curves represent orbital solutions from Table 1 .

Table 1. Circular orbital elements of WR 98 (WN7/WC+O8-9, $P=47.825 \mathrm{~d}$ ).

\begin{tabular}{lcccccc}
\hline \hline & element & N IV em. & N v em. & C III em. & He II em. & absorptions \\
\hline$V_{0}$ & $\left(\mathrm{~km} \mathrm{~s}^{-1}\right)$ & $-41 \pm 4$ & $-15 \pm 3$ & $-47 \pm 2$ & $4 \pm 2$ & $6 \pm 15$ \\
$K$ & $\left(\mathrm{~km} \mathrm{~s}^{-1}\right)$ & $106 \pm 6$ & $109 \pm 5$ & $72 \pm 3$ & $65 \pm 3$ & $112 \pm 16$ \\
$M_{\mathrm{WN} \sin ^{3} i}\left(\mathrm{M}_{\odot}\right)$ & $27 \pm 10$ & $28 \pm 10$ & $19 \pm 8$ & $18 \pm 7$ & \\
$M_{\text {OB }} \sin ^{3} i\left(\mathrm{M}_{\odot}\right)$ & $25 \pm 7$ & $27 \pm 7$ & $12 \pm 3$ & $10 \pm 3$ & \\
\hline \hline
\end{tabular}

that they are formed in the same stellar envelope (see Figure 1). We have also detected absorption lines upon the emissions. These absorptions correspond to a late 08-9 spectrum, and they move anti-phased with respect to the emission lines (see Figure 1). Thus WR 98 is a SB2. Orbital elements are listed in Table 1. The RV variations of absorption and emission lines indicate that $M_{\mathrm{WR}}^{\min }$ $\left(\simeq 28 \mathrm{M}_{\odot}\right)$ is similar or larger than $M_{\mathrm{O}}^{\min }\left(\simeq 27 \mathrm{M}_{\odot}\right)$.

\section{References}

Gamen, R.C., Niemela, V.S. 2002, New Astron. 7, 511

van der Hucht, K.A. 2001, New Astron. Reviews 45, 135

van der Hucht, K.A., Conti, P.S., Lundström, I., et al. 1981, Space Sci. Reviews 28, 227

Niemela, V.S. 1991, in: K.A. van der Hucht \& B. Hidayat (eds.), Wolf-Rayet Stars and Interrelations with Other Massive Stars in Galaxies, Proc. IAU Symp. No. 143 (Dordrecht: Kluwer), p. 201

Smith, L.F. 1968, MNRAS 138, 109

Smith, L.F., Shara, M.M., Moffat, A.F.J. 1996, MNRAS 281, 163 